\title{
Indoxyl sulfate induces renin release and apoptosis of kidney mesangial cells
}

\author{
Wei-Jie Wang ${ }^{1,2}$, Mei-Hua Cheng 3 , Mao-Feng Sun ${ }^{4,5}$, Sheng-Feng Hsu ${ }^{6,7}$ \\ and Ching-Sung Weng ${ }^{1,6}$ \\ 'Department of Biomedical Engineering, Chung Yuan Christian University, \\ No. 200, Chung Pei Road, Zhongli City, Taoyuan County, Taiwan \\ ${ }^{2}$ Division of Nephrology, Department of Internal Medicine, Taoyuan General Hospital, \\ Ministry of Health and Welfare, No 1492, Chung-Shan Road, Taoyuan City, Taoyuan County, Taiwan \\ ${ }^{3}$ Department of Rehabilitation, Taoyuan General Hospital, Ministry of Health and Welfare, \\ No 1492, Chung-Shan Road, Taoyuan City, Taoyuan County, Taiwan \\ ${ }^{4}$ School of Chinese Medicine, College of Chinese Medicine, China Medical University, \\ No.91 Hsueh-Shih Road, Taichung, Taiwan \\ ${ }^{5}$ Department of Acupuncture, China Medical University Hospital, No.91 Hsueh-Shih Road, Taichung, Taiwan \\ ${ }^{6}$ Graduate Institute of Acupuncture Science, College of Chinese Medicine, China Medical University, \\ No.91 Hsueh-Shih Road, Taichung, Taiwan \\ ${ }^{7}$ Department of Acupuncture, China Medical University Hospital, Taipei Branch, \\ No.91 Hsueh-Shih Road, Taichung, Taiwan
}

(Received April 26, 2014; Accepted June 23, 2014)

\begin{abstract}
Background: Indoxyl sulfate is considered to play a pathological role in the progression of chronic kidney disease. The aim of this study was to investigate the deleterious effects of indoxyl sulfate on kidney mesangial cells. Materials and Methods: Rat renal mesangial cells were exposed to indoxyl sulfate at a serial concentrations. Cytotoxicity of indoxyl sulfate on renal mesangial cells was determined using MTT assay. Protein levels of cleaved caspase-3, angiotensin, angiotensin converting enzyme (ACE) and renin were detected by immunoblotting. Reverse transcriptional PCR was performed to determine the mRNA expression. Results: Level of cleaved caspase- 3 was augmented while the cell viability was inhibited by indoxyl sulfate in a dose-dependent manner. The mRNA expressions of pro-renin and ACE were upregulated in mesangial cells exposed to indoxyl sulfate. Level of renin and ACE was increased in response to indoxyl sulfate exposure in time-dependent fashion. Conclusion: Indoxyl sulfate increased viability and induced cell death of renal mesangial cells, which is time-dependent. The loss of cell viability is attributed to caspase-3 activity through apoptosis pathway. RAS in renal mesangial cells is activated in response to indoxyl sulfate treatment.
\end{abstract}

Key words: Indoxyl sulfate, Apoptosis, CKD, Kidney mesangial cell, Renin-angiotension-system

\section{INTRODUCTION}

Chronic kidney disease (CKD) is characterized by a progressive deterioration of kidney function that can lead to kidney failure known as end-stage renal disease (ESRD). It has been reported to be a risk factor for cardiovascular disease and to contribute to diabetes-related cardiovascular complications (Jaradat and Molitoris, 2002). CKD-related hypertension is classified into 2 groups, volume-dependent and volume-independent, based on the response to diuresis or ultrafiltration (Sica and Carl, 2005). The latter involves an activation of renin-angiotensin systems (RAS) (Santos et al., 2012). On the other hand, high blood pressure has been reported to have a strong association with the risk of both diabetic and non-diabetic renal function decline or ESRD. Elevated systemic blood pressure is shown to cause mechanical stretch of glomerular capillaries, leading to damages and repair responses of renal microvasculature (Ofstad and Iversen, 2005). Repeated injury and repair of glomerular capillaries results in glomeruloscalerosis and proteinuria (Nishio et al., 2002; Ishida et al., 1999). It is evident

Correspondence: Ching-Sung Weng (E-mail: mrwwj.tw@yahoo.com.tw) 
that RAS plays a role in pathophysiology of hypertension and organ injury by regulating arterial pressure and sodium homeostasis. Recent studies have shown that aberrant activation of intrarenal RAS contributes to the development and progression of CKD and hypertension (Kagami, 2012; Wen et al., 2010). All components of RAS have been reported to be expressed in various cell types of kidney (Kobori et al., 2007). Proximal renal tubular cells express RAS components in response to uremic acid exposure (Sun et al., 2012). Treatment of mesangial cell with $\mathrm{H}_{2} \mathrm{~S}$ leads to an activation of RAS through reactive oxygen species (ROS) (Xue et al., 2013). It is suggested that intrarenal RAS could be activated in response to ROS generation.

Indoxyl sulfate is a metabolite of dietary protein resulting from the metabolism of bacterial indole in the liver. It is normally cleared by the kidney and then excreted into urine. As indoxyl sulfate level is associated with kidney function deterioration, it has been considered as a putative uremic toxin (Niwa, 2010b). Accumulation of indoxyl sulfate is found in patients with chronic kidney disease as a result of deteriorating renal function and impaired glomerular filtration (Niwa, 2010a). In turn, excessive serum indoxyl sulfate causes aberrant proliferation and fibrotic damages (Shimizu et al., 2012, 2013b). Nephrotoxicity of indoxyl sulfate has been demonstrated to involve a broad range of cellular responses. It has been reported that indoxyl sulfate induces production of ROS, which in turn activate nuclear factor$\kappa \mathrm{B}(\mathrm{NF}-\kappa \mathrm{B})$ and p53 in renal tubular cells (Shimizu et al., 2012). Moreover, exposure to indoxyl sulfate leads to an induction of senescence in a variety of cells, including nephrovascular cells (Adelibieke et al., 2012). Recent studies have reported that epithelial-to-mesenchymal transition (EMT) is suggested to be involved in the development and aggravation of CKD (Sun et al., 2012). Although endothelial and tubular cells are commonly used to study CKD, the renal mesangial cell providing structural support for glomerular capillary loops plays a role in progression of CKD in response to toxic injury. The mesangial cell responds to toxic challenges in several ways, including proliferation, release of proinflammatory cytokine, and fibrotic responses (Gelasco and Raymond, 2006). However, the effects of indoxyl sulfate on native glomerular cells such as mesangial cells remain sketchy.

The present study was focused on the cellular responses of kidney mesangial cell to indoxyl sulfate. We hypothesized that indoxyl sulfate could cause cell death through induction of apoptosis. In addition, activation of the intrarenal RAS in kidney mesangial cells was studied.

\section{MATERIALS AND METHOD}

\section{Cell culture}

Rat kidney mesangial cell line (CRL-2573, ATCC) was purchased from American Type Culture Collection and cultured in Dulbecco's modified Eagle's medium (DMEM; Gibco-BRL, Basel, Switzerland) supplemented with $2 \%$ (vol./vol.) fetal calf serum (FCS; Gibco-BRL). Cells in this experiment were used within three to six passages and were examined to ensure cell exhibiting the characteristics of kidney mesangial cells.

For investigation of protein expression, cells were seeded at a density of $5 \times 105 / \mathrm{ml}$ in a 6 -well plate and incubated with $500 \mu \mathrm{M}$ indoxyl sulfate (Sigma, St. Louis, MO, USA) for designated tmess. For investigation of ACE activity, cells were seeded at a density of $1 \mathrm{x}$ $10^{6} / \mathrm{ml}$ and exposed to $500 \mu \mathrm{M}$ indoxyl sulfate for indicated times.

\section{Cell viability}

Cell viability was determined using MTT assay. The MTT assay depends on the extent to which viable cells convert MTT bromide to an insoluble colored formazan product that can be determined spectrophotometrically. Cells were seeded in 96-well plate at a density of $5 \times 10^{5} / \mathrm{ml}$ and incubated overnight to allow cells to adhere. Cells were exposed to a serial concentration of indoxyl sulfate $(0,250,500$ and $1,000 \mu \mathrm{M})$ for the designated time period. After exposure, cells were washed with PBS twice and $200 \mu \mathrm{l}$ of DMEM without phenol red, containing $5 \mathrm{mg} / \mathrm{ml}$ MTT, was added to each well. Three hours later, the medium was aspirated, and the converted dye was solubilized within isopropanol $(0.1 \mathrm{~N} \mathrm{HCl}$ in isopropanol). The resulting absorbance from each well was measured at a wavelength of $570 \mathrm{~nm}$ with background subtraction at $630 \mathrm{~nm}$.

\section{Annexin V-PI staining}

An Annexin V (FITC-conjugated) apoptosis kit (BioVision, Mountain View, CA, USA) was used to analyze apoptotic cells. The experimental protocol followed the manufacturer's instructions. Cells $\left(5 \times 10^{5}\right)$ were cultured in 6-well plates and treated with $500 \mu \mathrm{M}$ indoxyl sulfate for 4, 8, 24 and $48 \mathrm{hr}$. Cells were trypsinized and washed with ice-cold PBS, which contained $0.5 \mathrm{ml}$ binding buffer, $5 \mu \mathrm{l}$ Annexin V-FITC, and $10 \mu \mathrm{l}$ propidium iodide PI. After incubation for $15 \mathrm{~min}$ in the dark, fluorescent micrographs of the cells were taken or cells were resuspended for the flow cytometric analysis (Beckton Dickinson, Franklin Lakes, NJ, USA). The mean values of the fluorescent intensities of FITC were determined 
using an FL-1 channel (488/530 ex/em nm).

\section{Immunoblotting}

Cells were washed twice with ice-cold PBS, and incubated with lysis buffer (Cell Signaling Technology, Beverly, MA, USA). The cell lysates were centrifuged at 14,000 g for $10 \mathrm{~min}$ at $4{ }^{\circ} \mathrm{C}$ and the resulting supernatants were retained. Protein concentrations were measured using $\mathrm{BCA}^{\mathrm{TM}}$ protein assay kit (Pierce, Rockford, IL, USA). Protein samples were subjected to $12.5 \%$ SDS-polyacrylamide gel, and transferred onto a PVDF membrane (Millipore, Bedford, MA, USA). The blotted membrane was blocked with $5 \% \mathrm{w} / \mathrm{v}$ skimmed milk in phosphate-buffered saline for $1 \mathrm{hr}$ followed by an incubation with $1 / 1,000$ dilution of the primary antibodies for $2 \mathrm{hr}$. The resulting membrane was incubated with $1 / 2,000$ dilution of peroxidase-conjugated secondary antibodies (Abcam, Cambridge, MA, USA) and bands were revealed using an enzyme-linked chemiluminescence detection kit (Amersham Biosciences, Piscataway, NJ, USA)

\section{Angiotensin converting enzyme (ACE) assay}

The ability to cleave the fluorogenic substrate, 7-McaYVADAPK (Dnp) (R\&D Systems, Minneapolis, MN, USA), by purified ACE was used to assess the respective activity of ACE in the culture. Cleavage of this substrate by either enzyme removes the 2,4-dinitrophenyl moiety that quenches the fluorescence of the 7-methoxycoumarin moiety, thus resulting in increased fluorescence.

\section{Reverse transcriptional PCR}

Total RNA was extracted through cell lysing using the RNeasy kit according to the manufacturer's instructions (Qiagen, Valencia, CA, USA). The purified RNA was used as a template to generate first-strand cDNA synthesis using RevertAid ${ }^{\mathrm{TM}}$ First Strand cDNA Synthesis Kit (Fermentas Life Sciences, St Leon-Rot, Germany). The correct size of the PCR products was checked by agarose gel electrophoresis. The primers used were listed in Table 1.

\section{Statistical analyses}

The value of each treatment group was presented as a mean with the standard deviation of triplicates. The data were compared using the One way ANOVA with TukeyKramer multiple comparisons post-test. The differences were considered significant for $p$ values less than 0.05 .

\section{RESULTS}

\section{Indoxyl sulfate induced cytotoxicity in mesnagial cells}

The cytotoxicity of indoxyl sulfate in kidney mesangial cells was determined using MTT assay. Kidney mesangial cells were exposed to indoxyl sulfate at concentrations of $0,250,500$ and $1,000 \mu \mathrm{M}$ for different periods of time. Increased viability was observed in the kidney mesangial cells exposed to 250 and $500 \mu \mathrm{M}$ of indoxyl sulfate for $12 \mathrm{hr}$, whereas $1,000 \mu \mathrm{M}$ of indoxyl sulfate had no influence on kidney mesangial cells (Fig. 1A). Cytotoxic effect of indoxyl sulfate was shown in the cells after a prolonged incubation for $24 \mathrm{hr}$ with a concentration of $1,000 \mu \mathrm{M}$ (Fig. 1B). A significant decrease of the cell viability was witnessed in prolonged cultures of kidney mesangial cells with indoxyl sulfate at concentrations of 500 and $1,000 \mu \mathrm{M}$. Treatment with $1,000 \mu \mathrm{M}$ of indoxyl sulfate for 48 and $72 \mathrm{hr}$ inhibited of cell viability to $71.63 \pm 1.68 \%$, and to $53.13 \pm 1.01 \%$, respectively (Figs. $1 \mathrm{C}$ and $1 \mathrm{D}$ ). Witnessing inhibition of viability, we next investigated the mechanism underlying the cytotoxic effect of indoxyl sulfate on kidney mesangial cells. We observed that the level of cleaved caspase 3 was elevated in response to indoxyl sulfate exposure (Fig. 2A). The apoptosis rates of kidney mesangial cells exposed to indoxyl sulfate 500 $\mu \mathrm{M}$ for designated time were determined through Annexin V-PI double staining by flow cytometry. Exposure to indoxyl sulfate for various incubation periods resulted in distinct apoptosis rate at $8 \%, 19 \%, 27 \%$, and $39 \%$ in HT29 cells, respectively.

\section{Indoxyl sulfate induces the production of renin and ACE in kidney mesangial cells}

It is evident that mesangium plays a deleterious role

Table 1. Primer sequences

\begin{tabular}{lll}
\hline Gene & Forward & Reverse \\
\hline ACE & 5'-CTGGAGACCACTCCCATCCTTTCT-3' & 5'-GATGTGGCCATCACATTCGTCAGAT-3' \\
Prorenin & 5'-CCACCCCAAACCTTCAAAGTCG-3' & 5'-TGCCCACAACCCCATCAAACTC-3' \\
Angiotensinogen & 5'-TGTTGCTGCTGAGAAGATTG-3' & 5'-CCGAGAAGTTGTCCTGGATG-3' \\
GAPDH & 5'-GATGGCATGGACTGTGGTCA-3' & 5'-GCAATGCCTCCTGCACCACC-3' \\
\hline
\end{tabular}


A
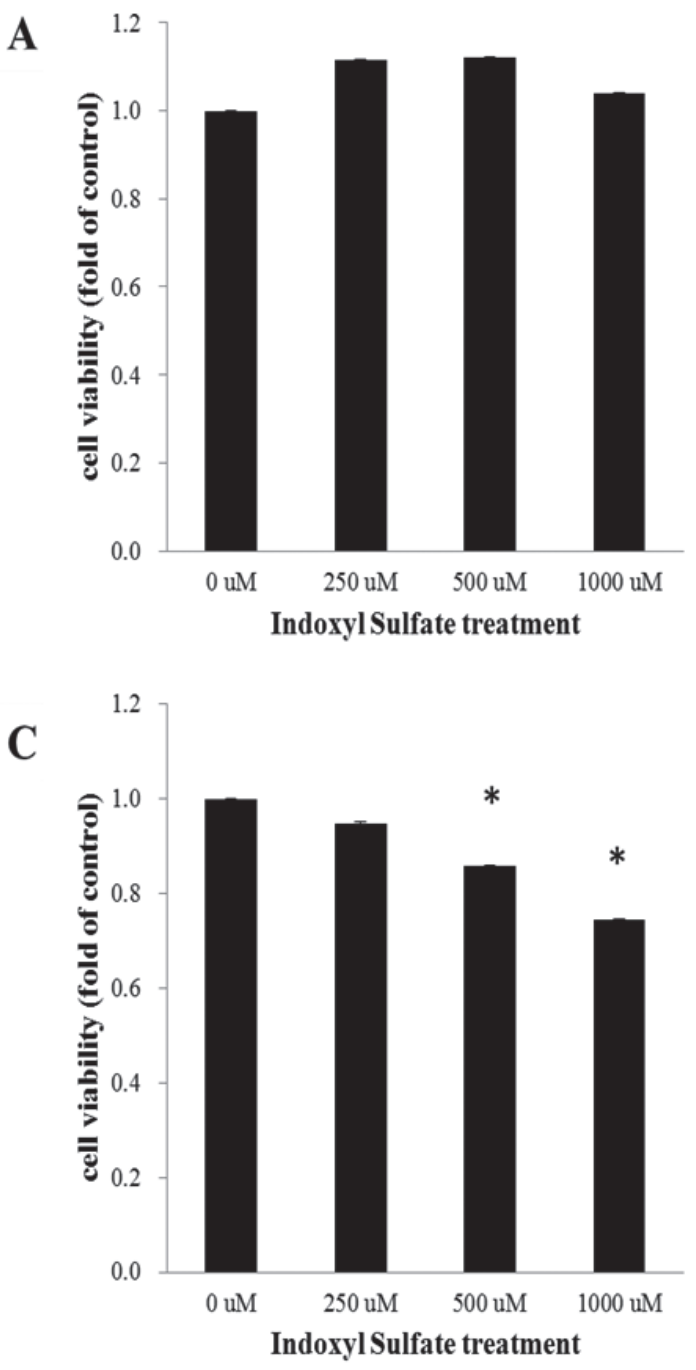
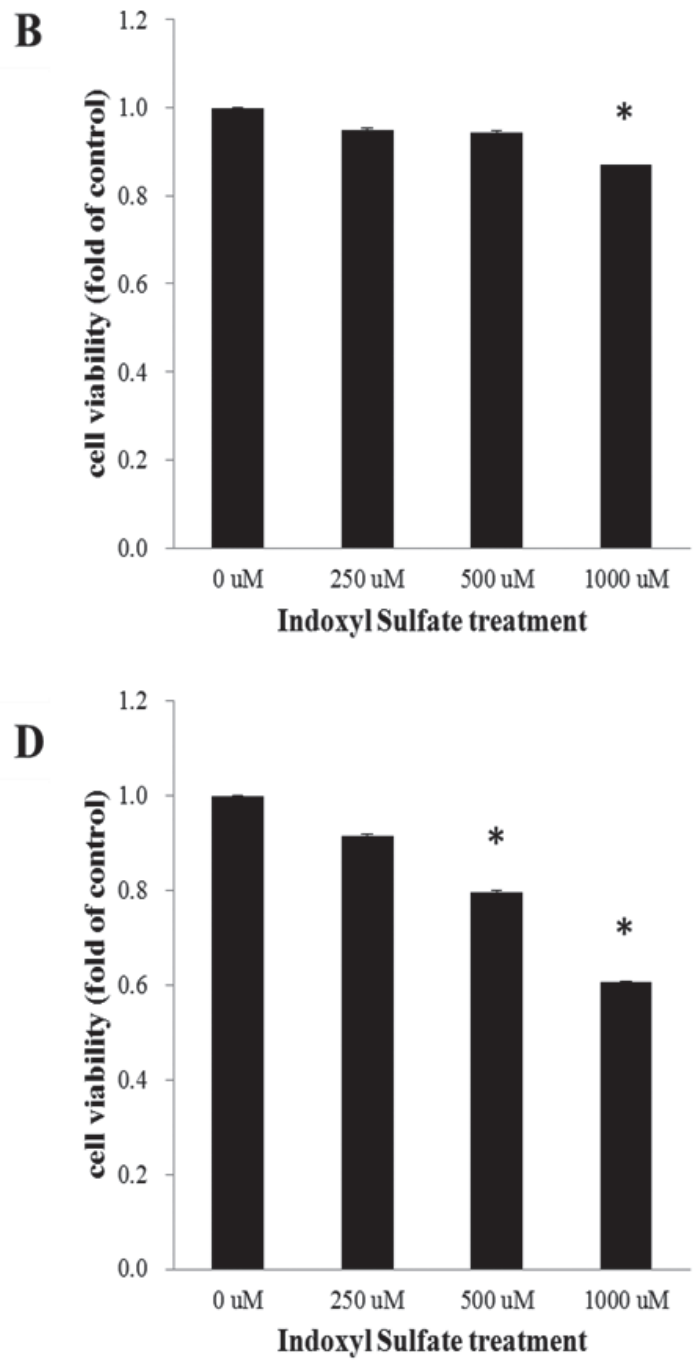

Fig. 1. Cytotoxic effects of indoxyl sulfate on kidney mesangial cells. Cells were treated with 250, 500, 1,000 $\mu \mathrm{M}$ indoxyl sulfate for (A) $12 \mathrm{hr}$, (B) $24 \mathrm{hr}$, (C) $48 \mathrm{hr}$, and (D) $72 \mathrm{hr}$. The cell survival was measured by MTT assay. Results were obtained from 3-5 experiments, and the bars represent the ratio \pm S.E. $(*: \mathrm{P}<0.01)$.

in the pathogenesis of malignant hypertension in response to toxic stress (Efrati et al., 2007). We hypothesized that indoxyl sulfate stimulates renin release and ACE production in kidney mesangial cell in CKD setting. Our data showed that indoxyl sulfate upregulated the mRNA expression of ACE in kidney mesangial cells at a concentration of $500 \mu \mathrm{M}$ in time-dependent fashion. A significant increase in mRNA level of pro-renin was detected in the cells treated with $500 \mu \mathrm{M}$ indoxyl sulfate for $24 \mathrm{hr}$; whereas pro-renin expressions were relatively low in the group of 1,000 $\mu \mathrm{M}$ (Fig. 3). Indoxyl sulfate had no effect on the mRNA expression of angiotensino gene. We next determined the changes in protein levels of ACE and ren- in in response to indoxyl sulfate exposure. The results showed that indoxyl sulfate induced an increase in protein level of ACE in kidney mesangial cells in a time-dependent manner (Fig. 4). The expression of renin was activated by indoxyl sulfate and reached a peak at the 12-hr time point. We also determined the level of secreted ACE by measuring the cleavage activity of ACE. The results showed that the cleavage activity of ACE was gradually increased over time in the cells treated with $500 \mu \mathrm{M}$ indoxyl sulfate (Fig. 5). Unexpectedly, secretion of ACE induced by $1,000 \mu \mathrm{M}$ of indoxyl sulfate did not follow the trends as that of the group of $500 \mu \mathrm{M}$ indoxyl sulfate. 
Indoxyl sulfate activates RAS

(A)

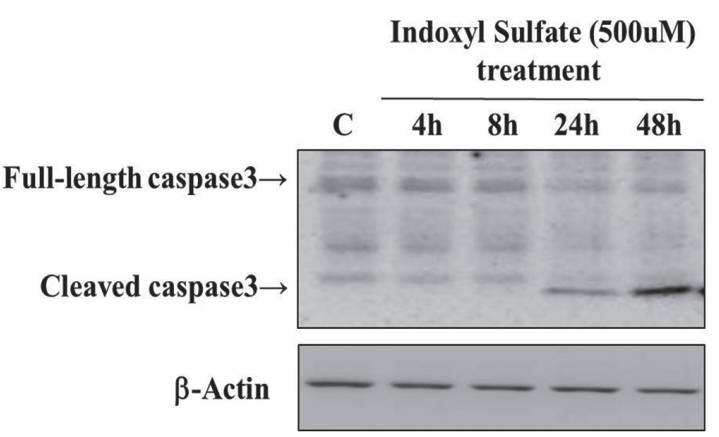

(B)

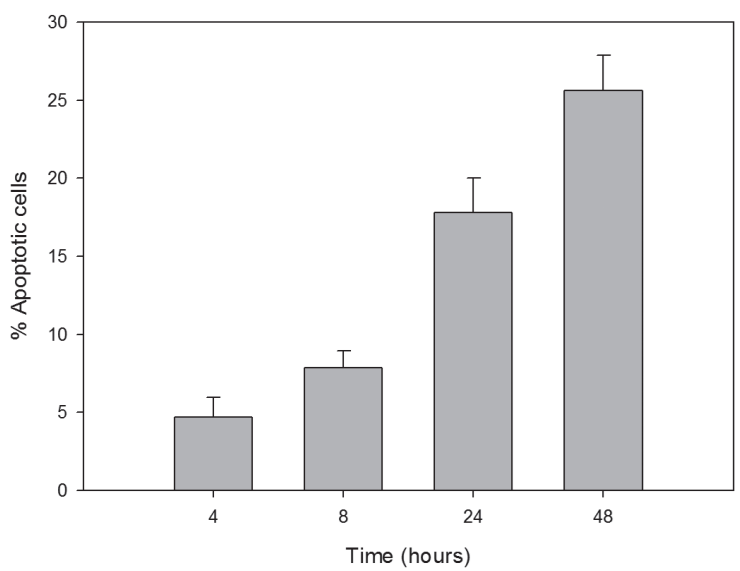

Fig. 2. Indoxyl sulfate induces apoptosis in kidney mesangial cells. (A) Changes in the expression of caspase 3 protein in response to $500 \mu \mathrm{M}$ indoxyl sulfate were analyzed using immunobloting. (B) Flow cytometry was done on the cells treated with indoxyl sulfate for designated times after fluorescein staining of anti-annexin $\mathrm{V}$ monoclonal antibody binding $(\mathrm{FL} 1-\mathrm{H})$ and propidium iodide uptake (FL3-H). Results are from a representative experiment of three replicates.

\section{DISCUSSION}

In the present study, we showed that indoxyl sulfate increased cell viability after $12 \mathrm{hr}$ of exposure and exerted cytotoxic effects in prolonged cultures. The inhibitory effects of indoxyl sulfate on kidney mesangial cells were found to involve activation of caspase- 3 and apoptosis. Moreover, kidney mesangial cells exhibited an increased expression of ACE and renin in response to prolonged exposure of indoxyl sulfate.

Accumulating evidence has demonstrated that indoxyl sulfate induces ROS generation in a variety of cells, including renal cells (Shimizu et al., 2013a; Niwa, 2010b). It is reported that indoxyl sulfate stimulates the

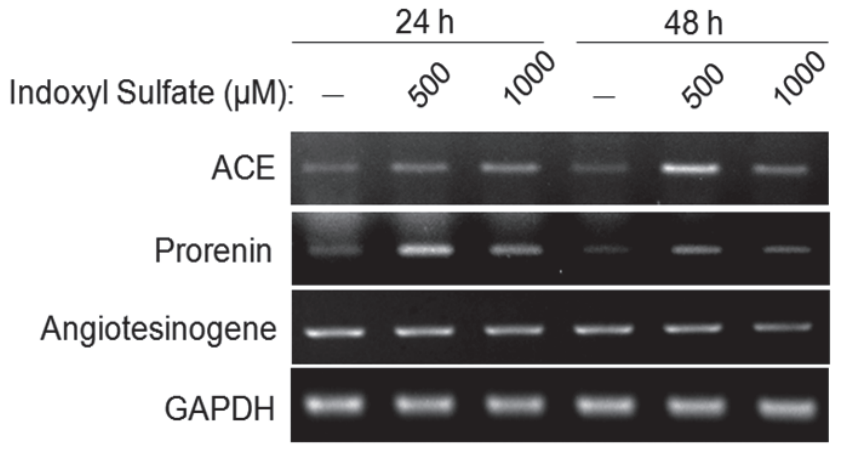

Fig. 3. Indoxyl sulfate activated the gene expression of renin-angiotensin-system in rat kidney mesangial cells. Polymerase chain reaction analysis shows that kidney mesangial cells had significantly increased renin and ACE mRNA expression.

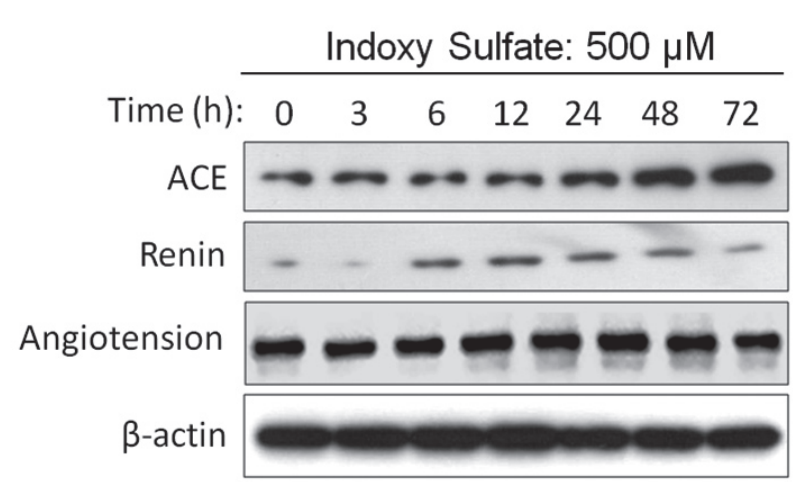

Fig. 4. Indoxyl sulfate activated the protein expression of renin-angiotensin- system in rat kidney mesangial cells. Immunoblotting shows that kidney mesangial cells treated with indoxyl sulfate at concentrations of 500 and $1,000 \mu \mathrm{M}$ had significantly increased renin and ACE protein expression.

production of intracellular and extracellular ROS in rat mesangial cells through NADPH oxidase (Gelasco and Raymond, 2006). It has been reported that indoxyl sulfate-induced ROS activate NF- $\mathrm{KB}, \mathrm{p} 65$, and $\mathrm{p} 53$ expression, leading to senescence induction in proximal tubular cells (Niwa and Shimizu, 2012; Adelibieke et al., 2012; Adijiang et al., 2011). Moreover, the cytotoxic effect of indoxyl sulfate has been revealed to involve apoptosis induction in rat renal tubular cells through an activation of ERK1/2 and p38 MAPK (Adelibieke et al., 2013). A recent study has shown that indoxyl sulfate induces AKT dephosphorylation leading to inhibited cell growth (Lin et al., 2013). Our result showed that that indoxyl sulfate increased the viability of kidney mesangial cell after $12 \mathrm{hr}$ 
W.-J. Wang et al.

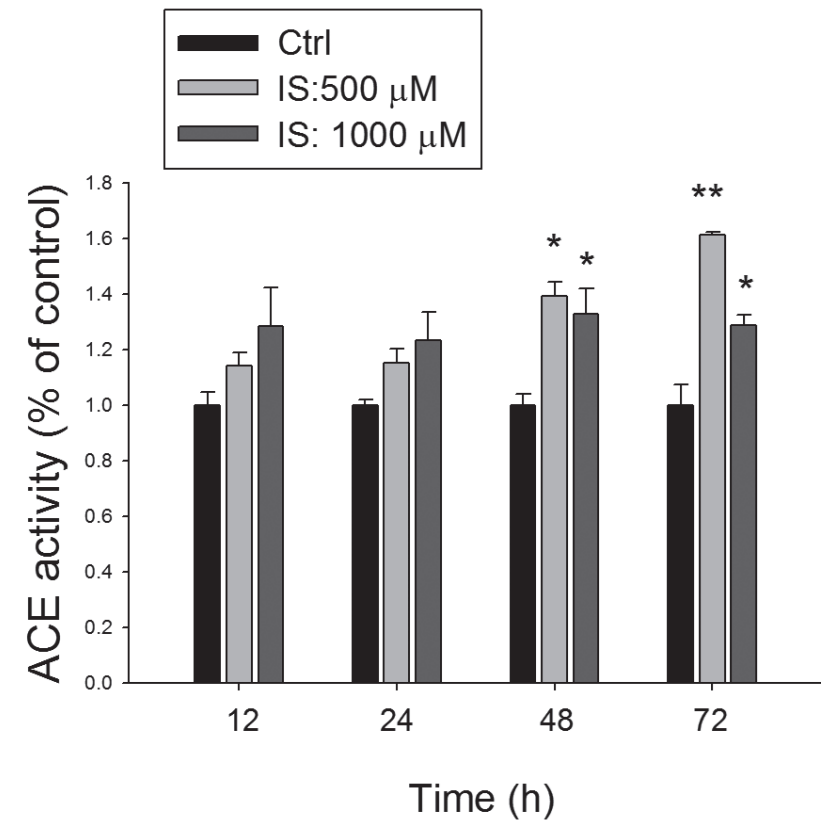

Fig. 5. Indoxyl sulfate enhance the ACE secretion in rat kidney mesangial cells. The ACE concentrations in the culture medium were measured by ELISA method. The ACE concentration of control cells was lower than detection limit. Treatment with indoxyl sulfate $(500$ and $1,000 \mu \mathrm{M})$ for indicated time significantly increased ACE production (*: $\mathrm{P}<0.05 ; * *$ : $\mathrm{P}<0.01$, vs. control). Ctrl: control, IS: Indoxy sulfate.

of incubation, suggesting that ROS induced by indoxyl sulfate have proliferative effect on kidney mesangial cells in a short-term incubation. A previous study in which mesangial cell was treated with gentamicin reported a similar phenomenon (Martinez-Salgado et al., 2004). Consistent with previous reports, prolonged exposure to indoxyl sulfate leads to augmented level of intracellular ROS and consequently cell death. Our data showed that indoxyl sulfate inhibited cell viability of kidney mesangial cells through the caspase- 3 activation. A set of experiments are required to elucidate the caspase- 3 apoptotic pathway involved.

The renin-angiotensin system is reported to contribute to the pathogenesis and progression of diabetic and nondiabetic CKD. In the classic pathway of the RAS, renin is secreted from the juxtaglomerular apparatus of the kidney and acts on the angiotensinogen to generate angiotensin I (Gilbert et al., 1999; Schunkert et al., 1991). Angiotensin I is converted in the lungs by ACE to angiotensin II. Angiotensin II acts on the heart and the kidneys by binding to the $\mathrm{G}$ protein-coupled receptors type 1 and type 2 (Siragy, 2010; Wolf and Neilson, 1996). The type 1 receptor mediates the more deleterious effects of angiotensin II - that is, vasoconstriction and cardiac and vessel hypertrophy angiotensin II stimulates tubular cells to mesenchymal myofibroblast transition, interstitial fibroblast proliferation, and ECM production. In renal glomerulus, the activation of RAS has been shown to induce apoptosis leading to loss of endothelial cells and podocytes (Stitt-Cavanagh et al., 2009; Liebau et al., 2006), whereas it promotes proliferation of mesangial cells. Mesangial cells play a role in maintaining the normal structure and functions of glomeruli. In physiology, renal mesangial cell exhibits the target of angiotensin II and the expression of all elements in RAS. The over-activation of RAS was associated with the functional changes of mesangial cells, including stimulating cell proliferation, TGF- $\beta$ and ECM productions (Cortes et al., 1999; Hori et al., 1998). It has been hypothesized that there is interaction between RAS and ROS. Angiotensin II stimulates ROS production by activating AT1 receptor (Chen et al., 2005). ROS is recently considered as a downstream signaling molecule of angiotensin II. In the present study, we showed that indoxyl sulfate induced augmented expression of RAS components, ACE and renin, in kidney mesangial cells. It is indicated that renin release upon indoxyl sulfate exposure may play a role in deterioration of renal function through paracrine RAS. As indoxyl sulfate activate ROS production, our finding is in agreement with previous research that activation of RAS leads to ROS generation.

In conclusion, indoxyl sulfate increased viability and induced cells death of kidney mesangial cell, which is time-dependent. The loss of cell viability is attributed to caspase- 3 activity through apoptosis pathway. RAS in kidney mesangial cells is activated in response to indoxyl sulfate treatment. The RAS components expressed include $\mathrm{ACE}$ and renin. Further study is necessary to explore role of kidney mesangial cells in indoxyl sulfate-induced renal damage and complications.

\section{REFERENCES}

Adelibieke, Y., Shimizu, H., Muteliefu, G., Bolati, D. and Niwa, T. (2012): Indoxyl sulfate induces endothelial cell senescence by increasing reactive oxygen species production and p53 activity. J. Ren. Nutr., 22, 86-89.

Adelibieke, Y., Shimizu, H., Saito, S., Mironova, R. and Niwa, T. (2013): Indoxyl sulfate counteracts endothelial effects of erythropoietin through suppression of Akt phosphorylation. Circ. J., 77, 1326-1336.

Adijiang, A., Shimizu, H., Higuchi, Y., Nishijima, F. and Niwa, T. (2011): Indoxyl sulfate reduces klotho expression and promotes senescence in the kidneys of hypertensive rats. J. Ren. Nutr., 21, 105-109. 
Indoxyl sulfate activates RAS

Chen, T.H., Wang, J.F., Chan, P. and Lee, H.M. (2005): Angiotensin II stimulates hypoxia-inducible factor 1 alpha accumulation in glomerular mesangial cells. Ann. N Y Acad. Sci., 1042, 286293.

Cortes, P., Riser, B.L., Yee, J. and Narins, R.G. (1999): Mechanical strain of glomerular mesangial cells in the pathogenesis of glomerulosclerosis: clinical implications. Nephrol. Dial. Transplant., 14, 1351-1354.

Efrati, S., Berman, S., Goldfinger, N., Erez, N., Averbukh, Z., Golik, A., Rotter, V. and Weissgarten, J. (2007): Enhanced angiotensin II production by renal mesangium is responsible for apoptosis/ proliferation of endothelial and epithelial cells in a model of malignant hypertension. J. Hypertens., 25, 1041-1052.

Gelasco, A.K. and Raymond, J.R. (2006): Indoxyl sulfate induces complex redox alterations in mesangial cells. Am. J. Physiol. Renal. Physiol., 290, F1551-1558.

Gilbert, R.E., Wu, L.L., Kelly, D.J., Cox, A., Wilkinson-Berka, J.L., Johnston, C.I. and Cooper, M.E. (1999): Pathological expression of renin and angiotensin II in the renal tubule after subtotal nephrectomy. Implications for the pathogenesis of tubulointerstitial fibrosis. Am. J. Pathol., 155, 429-440.

Hori, Y., Katoh, T., Hirakata, M., Joki, N., Kaname, S., Fukagawa, M., Okuda, T., Ohashi, H., Fujita, T., Miyazono, K. and Kurokawa, K. (1998): Anti-latent TGF-beta binding protein-1 antibody or synthetic oligopeptides inhibit extracellular matrix expression induced by stretch in cultured rat mesangial cells. Kidney Int., 53, 1616-1625.

Ishida, T., Haneda, M., Maeda, S., Koya, D. and Kikkawa, R. (1999): Stretch-induced overproduction of fibronectin in mesangial cells is mediated by the activation of mitogen-activated protein kinase. Diabetes, 48, 595-602.

Jaradat, M.I. and Molitoris, B.A. (2002): Cardiovascular disease in patients with chronic kidney disease. Semin. Nephrol., 22, 459473.

Kagami, S. (2012): Involvement of glomerular renin-angiotensin system (RAS) activation in the development and progression of glomerular injury. Clin. Exp. Nephrol., 16, 214-220.

Kobori, H., Nangaku, M., Navar, L.G. and Nishiyama, A. (2007): The intrarenal renin-angiotensin system: from physiology to the pathobiology of hypertension and kidney disease. Pharmacol. Rev., 59, 251-287.

Liebau, M.C., Lang, D., Bohm, J., Endlich, N., Bek, M., Witherden, I., Mathieson, P.W., Saleem, M.A., Pavenstadt, H. and Fischer, K. (2006): Functional expression of the renin-angiotensin system in human podocytes. Am. J. Physiol. Renal. Physiol., 290, F710-719.

Lin, C.Y., Hsu, S.C., Lee, H.S., Lin, S.H., Tsai, C.S., Huang, S.M., Shih, C.C. and Hsu, Y.J. (2013): Enhanced expression of glucose transporter-1 in vascular smooth muscle cells via the Akt/tuberous sclerosis complex subunit 2 (TSC2)/mammalian target of rapamycin (mTOR)/ribosomal S6 protein kinase (S6K) pathway in experimental renal failure. J. Vasc. Surg., 57, 475-485.

Martinez-Salgado, C., Eleno, N., Morales, A.I., Perez-Barriocanal, F., Arevalo, M. and Lopez-Novoa, J.M. (2004): Gentamicin treatment induces simultaneous mesangial proliferation and apoptosis in rats. Kidney Int., 65, 2161-2171.

Nishio, T., Haneda, M., Koya, D., Inoki, K., Maeda, S. and
Kikkawa, R. (2002): Cyclic AMP inhibits stretch-induced overexpression of fibronectin in glomerular mesangial cells. Eur. J. Pharmacol., 437, 113-122.

Niwa, T. (2010a): Indoxyl sulfate is a nephro-vascular toxin. J. Ren. Nutr., 20, S2-6.

Niwa, T. (2010b): Uremic toxicity of indoxyl sulfate. Nagoya J. Med. Sci., 72, 1-11.

Niwa, T. and Shimizu, H. (2012): Indoxyl sulfate induces nephrovascular senescence. J. Ren. Nutr., 22, 102-106.

Ofstad, J. and Iversen, B.M. (2005): Glomerular and tubular damage in normotensive and hypertensive rats. Am. J. Physiol. Renal. Physiol., 288, F665-672.

Santos, P.C., Krieger, J.E. and Pereira, A.C. (2012): Renin-angiotensin system, hypertension, and chronic kidney disease: pharmacogenetic implications. J. Pharmacol. Sci., 120, 77-88.

Schunkert, H., Ingelfinger, J.R. and Dzau, V.J. (1991): Evolving concepts of the intrarenal renin-angiotensin system in health and disease: contributions of molecular biology. Ren. Physiol. Biochem., 14, 146-154.

Shimizu, H., Bolati, D., Higashiyama, Y., Nishijima, F., Shimizu, K. and Niwa, T. (2012): Indoxyl sulfate upregulates renal expression of MCP-1 via production of ROS and activation of NF- $\mathrm{kB}$, p53, ERK, and JNK in proximal tubular cells. Life Sci., 90, 525530.

Shimizu, H., Saito, S., Higashiyama, Y., Nishijima, F. and Niwa, T. (2013a): CREB, NF-KB, and NADPH oxidase coordinately upregulate indoxyl sulfate-induced angiotensinogen expression in proximal tubular cells. Am. J. Physiol. Cell. Physiol., 304, C685-692.

Shimizu, H., Yisireyili, M., Higashiyama, Y., Nishijima, F. and Niwa, T. (2013b): Indoxyl sulfate upregulates renal expression of ICAM-1 via production of ROS and activation of NF- $\mathrm{KB}$ and p53 in proximal tubular cells. Life Sci., 92, 143-148.

Sica, D. and Carl, D. (2005): Pathologic basis and treatment considerations in chronic kidney disease-related hypertension. Semin. Nephrol., 25, 246-251.

Siragy, H.M. (2010): The angiotensin II type 2 receptor and the kidney. J. Renin. Angiotensin Aldosterone Syst., 11, 33-36.

Stitt-Cavanagh, E., MacLeod, L. and Kennedy, C. (2009): The podocyte in diabetic kidney disease. ScientificWorld Journal, 9, 1127-1139.

Sun, C.Y., Chang, S.C. and Wu, M.S. (2012): Uremic toxins induce kidney fibrosis by activating intrarenal renin-angiotensin-aldosterone system associated epithelial-to-mesenchymal transition. PLoS One, 7, e34026.

Wen, X., Murugan, R., Peng, Z. and Kellum, J.A. (2010): Pathophysiology of acute kidney injury: a new perspective. Contrib. Nephrol., 165, 39-45.

Wolf, G. and Neilson, E.G. (1996): From converting enzyme inhibition to angiotensin II receptor blockade: new insight on angiotensin II receptor subtypes in the kidney. Exp. Nephrol., 4 Suppl 1, 8-19.

Xue, H., Yuan, P., Ni, J., Li, C., Shao, D., Liu, J., Shen, Y., Wang, Z., Zhou, L., Zhang, W., Huang, Y., Yu, C., Wang, R. and Lu, L. (2013): H(2)S Inhibits Hyperglycemia-Induced Intrarenal ReninAngiotensin System Activation via Attenuation of Reactive Oxygen Species Generation. PLoS One, 8, e74366. 